\title{
Role of endoplasmic reticulum stress in depression (Review)
}

\author{
JIAXIN MAO ${ }^{1,2^{*}}$, YANRAN HU ${ }^{1,2^{*}}$, LIEMIN RUAN ${ }^{2}$, YUNXIN $\mathrm{JI}^{2}$ and ZHONGZE LOU ${ }^{2}$ \\ ${ }^{1}$ Department of Mental Health and Psychiatry, Medical School of Ningbo University, Ningbo, Zhejiang 315211; \\ ${ }^{2}$ Department of Psychosomatic Medicine, Ningbo First Hospital, Ningbo Hospital of Zhejiang University, \\ Medical School of Ningbo University, Ningbo, Zhejiang 315010, P.R. China
}

Received March 15, 2019; Accepted October 10, 2019

DOI: $10.3892 / \mathrm{mmr} .2019 .10789$

\begin{abstract}
Depression is a devastating mood disorder that causes profound disability worldwide. Despite the increasing number of antidepressant medications available, the treatment options for depression are limited. Therefore, understanding the etiology and pathophysiology of depression, and exploiting potential novel agents to treat and prevent this disorder are imperative. Endoplasmic reticulum (ER) stress activates the unfolded protein response and mediates the pathogenesis of psychiatric diseases, including depression. Emerging evidence in human and animal models suggests an intriguing link between ER stress and depression. The ER serves as an important subcellular organelle for the synthesis, folding, modification, and transport of proteins, a process that is highly developed in neuronal cells. Perturbations of ER homeostasis lead to ER stress, and ER stress helps to restore the normal ER function by restoring the protein-folding capacity of the ER. This biological defense mechanism is imperative to prevent the
\end{abstract}

Correspondence to: Dr Zhongze Lou or Ms. Yunxin Ji, Department of Psychosomatic Medicine, Ningbo First Hospital, Ningbo Hospital of Zhejiang University, Medical School of Ningbo University, 59 Liuting Street, Haishu, Ningbo, Zhejiang 315010, P.R. China

E-mail: lorenzo_87@163.com

E-mail: janegege123@163.com

*Contributed equally

Abbreviations: ER, endoplasmic reticulum; UPR, unfolded protein response; GRP78, 78-kDa glucose-regulated protein; GRP94, 94-kDa glucose-regulated protein; ERAD, ER-associated degradation; MDD, major depressive disorder; PERK, protein kinase R-like ER kinase; IRE1 $\alpha$, inositol-requiring enzyme $1 \alpha$; ATF6, activating transcription factor 6 ; eIF $2 \alpha$, eukaryotic translation initiation factor $2 \alpha$; ATF4, activating transcription factor 4 ; CHOP, CCAAT/enhancer-binding protein-homologous protein; XBP1, X-box binding protein 1; EDEM1, ER degradation-enhancing- $\alpha$ mannosidase-like 1; CUMS, chronic unpredictable mild stress; $\mathrm{H} 2 \mathrm{~S}$, hydrogen sulfide; CRS, chronic restraint stress; SSRI, selective serotonin reuptake inhibitor; Sig-1R, $\sigma-1$ receptor

Key words: depression, endoplasmic reticulum stress, apoptosis, unfolded protein response, $78-\mathrm{kDa}$ glucose-regulated protein disease. However, excessive or persistent ER stress eventually causes cell death. If the damage occurs in the hippocampus, the amygdala and striatum and other areas of the neurons will be involved in the development of depression. In this review article, we explore how ER stress might have an important role in the pathophysiology of depression and how different drugs affect depression through ER stress.

\section{Contents \\ 1. Introduction \\ 2. ER stress \\ 3. ER stress and depression \\ 4. ER stress and medicine \\ 5. Conclusions}

\section{Introduction}

Depression is a highly prevalent mood disorder characterized by depressed mood, declined interest and so-called autonomic function changes, including alterations in appetite, sleep, and energy levels (1). Depression affects many aspects of patients' lives, including social interaction, relationships with family, and working efficiency, and $>350$ million people worldwide battle with the disorder every day (2). Depression is one of the leading causes of disabling medical conditions worldwide, and $>60 \%$ of those who commit suicide suffer from the disease (3). Despite its tremendous burden, high prevalence, the extensive research over the last half-century, and the increasing number of antidepressant treatment options available, an efficient cure for depression is lacking.

The endoplasmic reticulum (ER) is an important subcellular organelle involved in the synthesis, post-translational modifications, and proper folding of secretory proteins, and calcium homeostasis (4). A variety of physiological conditions, including hypoxia, stress, hypoglycemia, calcium depletion, oxidative stress, and a high-fat diet, can disrupt the protein folding process and consequently result in the accumulation of unfolded and misfolded proteins in the ER, a condition termed ER stress (5). Under ER stress, the unfolded protein response (UPR) is activated to minimize the overloading caused by the unfolded proteins. However, if ER functions are severely damaged, apoptosis signals are triggered (6). 
Maintaining the normal function of the ER requires the help of ER chaperones, of which the 78-kDa glucose-regulated protein (GRP78) and 94-kDa glucose-regulated protein (GRP94) are important members (7). Many cellular processes require ER chaperone involvement, including the transfer of newly synthesized peptides on the ER membrane, the folding and assembly of proteins, the degradation of misfolded proteins by the ubiquitin-proteasome system, the autophagy-lysosomal system, and the regulation of calcium homeostasis, which act as stress sensors for the ER (8-11). Multiple studies have demonstrated that ER stress is associated with psychiatric disorders. Bown et al (12) revealed that levels of the ER stress proteins GRP78, GRP94 and calreticulin are elevated in the postmortem temporal cortex of patients with major depressive disorder (MDD), with Nevell et al (13) similarly demonstrating that systemic and persistent activation of ER stress is associated with MDD. Another study reported that stressful life events across the lifespan play an important role in disease etiology (14). Stress is one of the common causes of depressive episodes; sustained stress can also disrupt hippocampal nerve regeneration in adults, indirectly inhibit dopamine function, and exaggerate the amygdala's memory of negative stimuli (15). Therefore, it is hypothesized that ER stress may be a major culprit in the progression of depression. This review aimed to summarize the current knowledge of the role of ER stress in depression.

\section{ER stress}

ER stress refers to the molecular and biochemical changes in the homeostatic morphology and function of the ER upon the stimulation of cells by internal or external factors, which block the processing and transport of proteins, and lead to the accumulation of large amounts of unfolded or misfolded proteins in the ER (16). As a result, cells take corresponding measures to relieve ER stress and promote the recovery of normal ER function. Cellular survival is only achieved when the ER stress response resolves these stimuli and produces normal proteins (17). If residual unfolded proteins are released as mutants, specific apoptotic responses occur (18). To relieve ER stress and restore homeostatic functions within the ER on a cellular level, the UPR triggers three types of protective cellular responses depending on the cause of induction: The upregulation of genes encoding ER chaperones, the attenuation of translation, or the promotion of ER-associated degradation (ERAD) of aggregated proteins (19). These are adaptive responses to protein accumulation in the ER and relieve ER stress by reducing protein synthesis, promoting protein degradation, and increasing molecular chaperones that help protein folding. Protein kinase R-like ER kinase (PERK), inositol-requiring enzyme $1 \alpha($ IRE1 $\alpha)$, and activating transcription factor 6 (ATF6) are important transmembrane proteins that initiate UPR (20). In unstressed cells, these ER transmembrane signaling molecules are maintained in an inactive state by binding to GRP78 (21). Upon detecting ER stress, the UPR signaling pathway is triggered, and GRP78 is rapidly released from these three molecules and binds to unfolded proteins (Fig. 1) (22).

Following dissociation from GRP78, PERK homodimerizes to promote its autophosphorylation and activation (23).
PERK activation then triggers the phosphorylation of eukaryotic translation initiation factor $2 \alpha$ (eIF2 $\alpha$ ), which activates the expression of multiple transcription factors, such as activating transcription factor 4 (ATF4) (23). Phosphorylated eIF2 $\alpha$ activity is inhibited during the early stages of stress, which slows down the synthesis and translation of most proteins in the cell, and reduces the load of protein folding in the ER. In addition, ATF 4 serves a protective role by regulating amino acid metabolism, redox reactions and protein secretion (24). However, as the time and intensity of the stress response increases, activated ATF4 induces the expression of the pro-apoptotic CCAAT/enhancer-binding protein-homologous protein (CHOP) (25).

IRE1 $\alpha$ is a transmembrane protein containing serine/threonine kinase and endonuclease domain (26). Following the dissociation of IRE1 $\alpha$ from GRP78, IRE1 $\alpha$ is self-activated by homodimerization and phosphorylation of the intracytoplasmic domain, resulting in the allosteric activation of its surrounding endonuclease domain (24). Activated IRE1 $\alpha$ splices the 26-base intron in the mRNA precursor of X-box binding protein 1 (XBP1), which is induced by ATF6, and the spliced mRNA undergoes translational frame shifting to encode the steady-state transcription factor XBP1 (27). The transcription factor XBP1s not only induces the transcription of the GRP78 and CHOP genes but also activates the ERAD program through ER degradation enhancing $\alpha$ mannosidase-like 1 (EDEM1) in an attempt to restore ER homeostasis $(28,29)$. Therefore, XBP1s is crucial to restoring ER homeostasis.

In the unstressed state, ATF6 is mainly present in the ER as a zymogen, but under ER stress, it is induced to dissociate from GRP78 and transport into Golgi bodies. The ATF6 $\alpha$ transcription factor is released following cleavage by the Golgi site-1 and -2 proteases (30). Together with XBP1, ATF6 $\alpha$ activates the transcription of targets such as GRP78, thereby counteract ER stress and exert protective effects to promote cell survival (31). Collectively, these transduction pathways coordinate together to counteract ER stress through negative feedback, reducing the number of unfolded proteins and facilitating cell survival (17). Nonetheless, when the mechanism fails, a large amount of calcium is released into the cytoplasm to induce apoptosis, whilst persistent UPR signals induce chronic ER stress (18). The UPR actively promotes cell death through apoptosis following the activation of CHOP, JNKs, proapoptotic Bcl-2-like protein 11 (Bim; a member of the Bcl-2 family), and caspases (Fig. 2) (32).

\section{ER stress and depression}

Animal studies. Rats or mice exposed to chronic unpredictable mild stress (CUMS) are widely used as depression models (33). Previous results have suggested that the depressive behavior of CUMS mice is related to ER stress, and that the underlying mechanism is the insufficient synthesis of ATP, and the overactivated oxidative and ER stress which leads to neuronal apoptosis or death (34). Another previous study reported that CUMS-induced depression-like behavior in rats is due to the disturbance of hippocampal hydrogen sulfide $\left(\mathrm{H}_{2} \mathrm{~S}\right)$ generation, which in turn promotes hippocampal ER stress responses including the upregulation of GRP78, CHOP, 


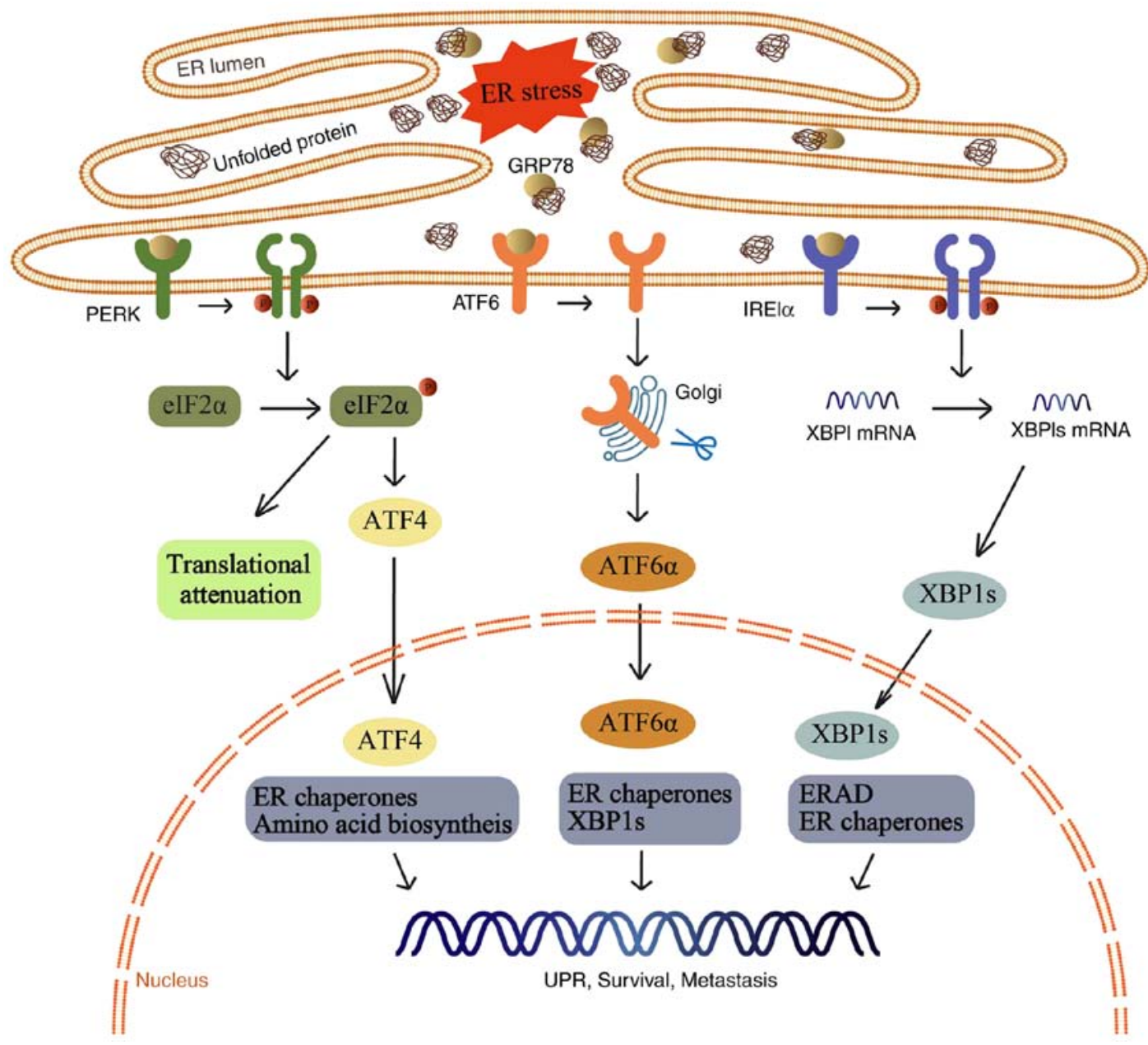

Figure 1. A variety of physiological conditions can disrupt the protein folding process and consequently result in the accumulation of unfolded and misfolded proteins in the ER, a condition termed ER stress. Under ER stress, the UPR is activated to minimize overloading. If the UPR is successful in reducing the number of unfolded and misfolded proteins, UPR signaling is attenuated and the cell survives. ER, endoplasmic reticulum; GRP78, 78-kDa glucose-regulated protein; PERK, protein kinase R-like ER kinase; eIF2 $\alpha$, eukaryotic translation initiation factor $2 \alpha$; ATF4, activating transcription factor 4; ATF6, activating transcription factor 6; IRE1 $\alpha$, inositol-requiring enzyme 1 $\alpha$; XBP1, X-box binding protein 1; ERAD, endoplasmic reticulum associated degradation; UPR, unfolded protein response.

and cleaved caspase-12 expression (35). $\mathrm{H}_{2} \mathrm{~S}$ is the third most prevalent endogenous signaling gasotransmitter and serves a key role in both neuromodulation and neuroprotection (36). Moreover, $\mathrm{H}_{2} \mathrm{~S}$ has been suggested to inhibit hippocampal ER stress by upregulating hippocampal silence signal regulator 1 , an essential metabolically regulated transcription factor, thereby attenuating depression-like behaviors in rats (37). These findings suggest that $\mathrm{H}_{2} \mathrm{~S}$ may provide a novel target for the treatment of depression.

Recent evidence has demonstrated that chronic restraint stress (CRS) induces cognitive impairment, and anxiety- and depression-like behavior in rodents (38). Previous findings by Jangra $e t$ al suggest that honokiol (a traditional Chinese medicine isolated from the bark of Magnolia officinalis tree) eliminates CRS-induced cognitive impairment and depressive-like behavior by preventing the elevation of GRP78 and CHOP in the hippocampus of mice (39). Furthermore, in CRS mice, the expression levels of GRP78 and CHOP in the hippocampus, and of CHOP in the prefrontal cortex, are significantly upregulated compared with those in the control group, while sodium phenylbutyrate (an ER stress inhibitor) and edaravone (a free radical scavenger) not only reverse the high expression of these genes, but also improve the cognitive deficits and depression-like behavior observed in the model mice (40). The expression levels of GRP78, ATF6, XBP1, CHOP, and growth arrest and DNA damage-inducible protein (a regulatory subunit of an eIF $2 \alpha$-specific phosphatase complex) are also increased in the CRS rat striatum, indicating that ER stress is involved in CRS-induced depression-like abnormal behaviors (41).

Lipopolysaccharide is an endotoxin that causes anxiety- and depression-like behavior in rodents after central or peripheral administration (42). Its administration is reported to significantly upregulate GRP78 mRNA expression in the hippocampus, suggesting that UPR is involved in lipopolysaccharide-evoked behavioral anomalies (43). Social defeat stress-vulnerable mice also demonstrate depression-like behavior, which is associated with a significant increase in the expression of GRP78, CHOP and choline acetyltransferase in the amygdala (44). Liu et al found that the expression of GRP78 and XBP1 were significantly increased in the hippocampus of mice with social defeat stress (45). Furthermore, chronic social defeat stress can activate the PERK-eIF2 $\alpha$ signaling pathway in the hippocampus, leading to the downregulation of brain-derived neurotrophic 


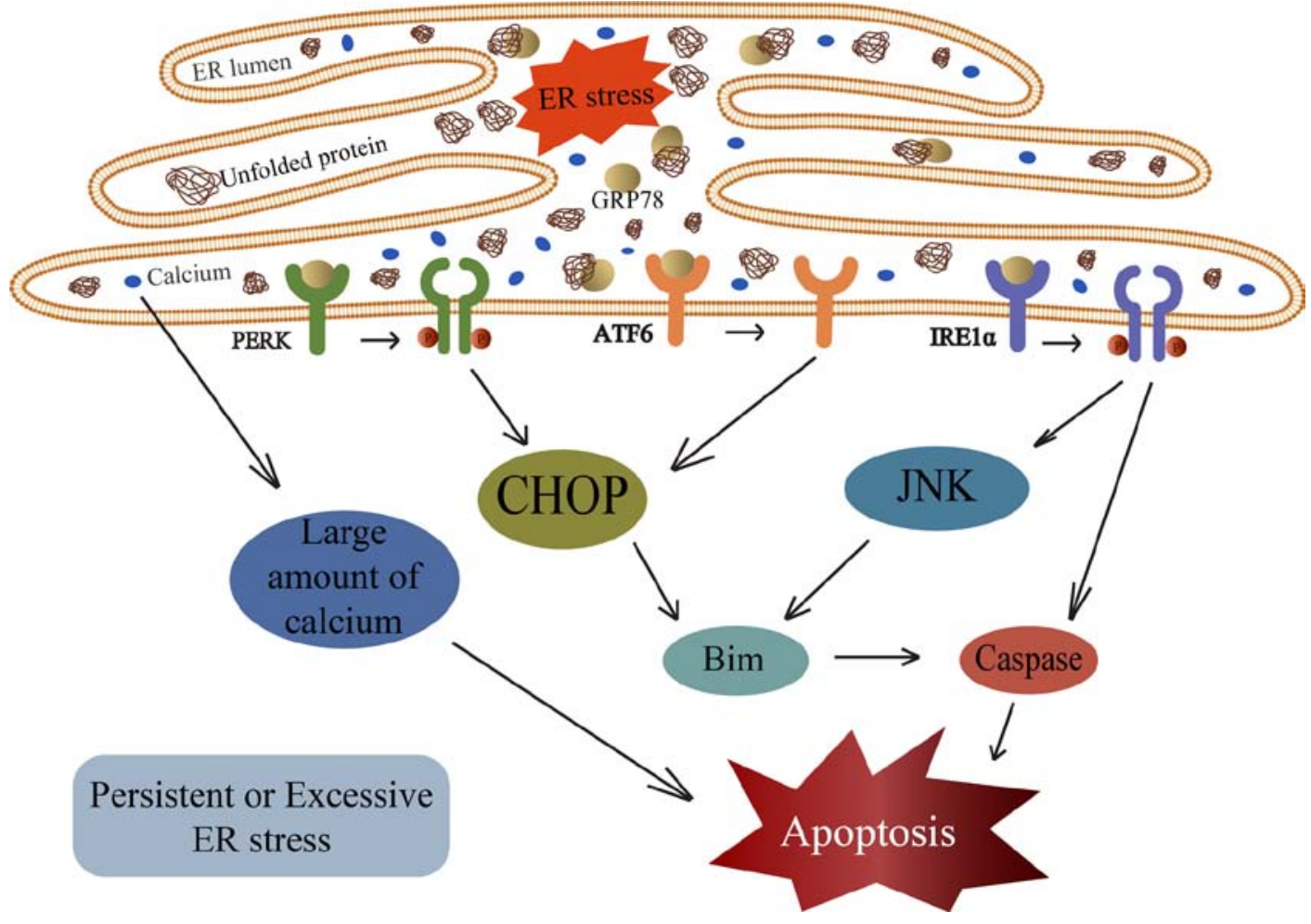

Figure 2. When the function of ER is severely impaired, a large amount of calcium is released into the cytoplasm to induce apoptosis. UPR also triggers apoptotic signals through CHOP, JNKs, Bim, and caspases. ER, endoplasmic reticulum; GRP78, 78-kDa glucose-regulated protein; PERK, protein kinase R-like ER kinase; ATF6, activating transcription factor 6; IRE1 $\alpha$, inositol-requiring enzyme 1 $\alpha$; CHOP, CCAAT/enhancer-binding protein-homologous protein; Bim, Bcl-2-like protein 11.

factor expression levels, and eventually depression-like behavior and memory impairment in mice (46). Sharma et al also demonstrated that inhibition of PERK expression in the hippocampus can enhance hippocampal-dependent memory and reverse memory deterioration in mice (47), suggesting that cognitive function can be improved by regulating the expression levels of PERK at the Cornu Ammon 1 region of the hippocampus. In addition, plasma levels of corticosterone and the expression of genes encoding GRP78, GRP94, ATF6, XBP1, ATF4, and CHOP increased in the hippocampus of rats with learned helplessness, suggesting that depression-like behavior may be associated with excessive persistence of ER stress (48). Spinal cord injury in rodent models causes depressive-like behavior and impairment of spatial memory retention (49-51). Experimental studies have reported that chronically activated ER stress in the brain and newly formed immature neurons in the subgranular area of the hippocampus may be detrimental to the survival and regeneration of impaired cognitive and depressive neurons following spinal cord injury (52). Finally, the introduction of an Alzheimer's patient-associated mutation in the gene encoding carboxypeptidase E/NF- $\alpha 1$ into transgenic mice leads to increased ER stress and decreased expression of a pro-survival protein, Blc-2, ultimately resulting in symptoms resembling dementia and depression (53). In summary, the above animal studies have demonstrated a link between ER stress and depression.

Clinical studies. To explore the hypothesis that ER stress has an important influence on the pathophysiology of depression in human studies, Behnke et al (8) measured the expression levels of GRP78, GRP94, and calreticulin in postmortem samples of psychiatric patients. Higher expression levels of GRP78, GRP94, and calreticulin were found in the temporal cortex of patients with MDD who died by suicide compared with MMD patients with non-suicide deaths or other groups (8). Although some of the patients with bipolar disorder or schizophrenia died by suicide, no such differences in the expression levels of these proteins were found in these patients (12). Furthermore, xanthine oxidase activity, which produces reactive oxygen species and is a central mechanism of oxidative stress, is increased in temporal lobe tissue of patients with recurrent depression $(54,55)$. In addition, upon analyzing the expression levels of GRP78, EDEM1, CHOP, and XBP1, the major indicators of the ER stress response, with reverse transcription-quantitative PCR using leukocyte-derived RNA samples from 86 participants in the Detroit Neighborhood Health Study, patients with MDD had significantly higher levels of GRP78, EDEM1, CHOP, and XBP1 compared to control patients (13). These data suggest that the ER stress response pathways in those MDD patients are indicative of sustained activation. Altogether, these studies have suggested that the ER stress response pathway is continuously activated in MDD patients and warrants further investigation.

\section{ER stress and medicine}

Herbal medicine. Previous studies have found that numerous compounds exert antidepressive properties that may be linked to their ability to affect ER stress. Chronic luteolin treatment results in an antidepressant-like effect by inhibiting apoptosis 
induced by hippocampal ER stress, leading to the increased expression of the genes encoding GRP78 and GRP94 and reduced cleavage of caspase-3 (56). By contrast, longistyline C exerts antidepressive properties by inhibiting the levels of GRP78, CHOP and XBP1, thus, relieving ER stress (57). Another previous study reported that the water extraction product of Panax ginseng possesses antidepressant-like activity in both acute and chronic stress models of depression, with the underlying molecular mechanism depending on the downregulation of ER stress-related proteins, such as CHOP, GRP78, XBP1, and caspase-12 (58). Saikosaponins may also exert antidepressant-like effects through their protective effects on neuronal cells, including their ability to downregulate GRP78, CHOP and XBP1 to stabilize ER stress and restore mitochondrial membrane potential activity to recover mitochondrial function (59). Finally, a recent study demonstrated that gastrodin can improve depression-like behavior in mice by inhibiting ER stress (60), and the mechanism by which Aralia elata treats depression after illness also involves ER stress (61).

Antidepressants. Fluvoxamine is a selective serotonin reuptake inhibitor (SSRI) with high affinity for the $\sigma-1$ receptor (Sig-1R) (62). In a leptin resistance study, Hosoi et al revealed that fluvoxamine attenuated ER stress through the Sig-1R (63). Sig-1R forms a complex with another molecular chaperone, such as GRP78, at the ER-mitochondrial interface to regulate calcium ion signaling and cell survival (64). Similarly, a previous study by Omi et al demonstrated that fluvoxamine-mediated upregulation of Sig-1R expression promoted neuroprotection by inhibiting ER stress-mediated apoptosis and increasing ATF4 translation (65). Finally, Terada et al reported that fluvoxamine improved depression-like behavior in mice following chronic dexamethasone infusions via the recovery of brain-derived neurotrophic factor/XBP1/Sig-1R/5-hydroxytryptamine 2A receptor signaling cascades (66).

Fluoxetine is a widely used SSRI in clinical practice that can prolong and increase the action of serotonin (67). Ma et al revealed that fluoxetine induced apoptosis through CHOP-dependent ER stress-related apoptotic pathways in glioma cells, such as the PERK/eIF2 $\alpha / \mathrm{ATF} 4 / \mathrm{CHOP}$ and ATF6/CHOP signaling pathways (68). In addition, desipramine promotes antitumor activity in glioma by inducing autophagy through the PERK/eIF2 $\alpha$ and ATF6 signaling pathways (69) and the rapid antidepressant effects of ketamine may also be related to ER stress (70). Notably, previous experimental studies suggest that liver damage caused by some drugs is also associated with excessive activation of ER stress. For example, the commonly used SSRI antidepressant sertraline leads to an increase in PERK, IRE1 $\alpha$, and CHOP expression levels (71) and nefazodone significantly increases CHOP, ATF4, and phosphorylated eIF2 $\alpha$ levels (72). In addition, a recent study demonstrated that the SSRI escitalopram significantly reduces the expression level of ER stress marker proteins, thereby exerting its antidepressant effect (73). To sum up, most studies suggest that antidepressants work by reducing ER stress.

Other. The Bax inhibitor 1 (BI-1) is an evolutionarily conserved ER protein that has an antiapoptotic effect on ER stress-induced cell death (74). Therefore, in the short term,
BI-1 may serve a protective role in the depression-like behaviors induced by chronic mild stress (75). In addition, previous findings by Hunsberger et al indicated that the expression of BI-1 was protective against anhedonia (76). Alternatively, evidence suggests that an estrogen receptor $\beta$ agonist could ameliorate ER stress-induced anxiety- and depression-like behavior through the IRE1 $\alpha /$ XBP1 pathway (77). Finally, in rats, a soluble epoxide hydrolase inhibitor mitigated the development of depression-like behaviors by alleviating ER stress (78).

\section{Conclusions}

Current research suggests that ER stress serves a key role in the pathophysiology of depression. ER stress-related proteins are significantly increased in animal models of depression, including within the hippocampus, prefrontal cortex, amygdala, and striatum, indicating that ER stress is involved in the pathogenesis of depression. Clinical studies are relatively limited, but evidence has revealed that patients with depression exhibit persistent activation of the ER stress system. This suggests that the treatment and prevention of depression should focus on ER stress-related signaling pathways. Future research should elucidate further the signaling pathways involved in depression-related ER stress. Furthermore, studies with antidepressant drugs are contradictory; herbal medicine and fluvoxamine inhibit the excessive activation of the ER stress system, whereas fluoxetine, desipramine, sertraline, and nefazodone potentiate ER stress. Although the latter can activate ER stress to promote cell apoptosis, no studies have definitely demonstrated that they cause neuronal apoptosis. Thus, the effects of these antidepressant drugs on different types of cellular ER stress systems should be explored in depth. Concurrently, the role of ER stress in depression should be further clarified from the perspective of gene transcription and translation. In addition, the activation of ER stress in animal models of depression is often accompanied by the occurrence of oxidative stress and inflammation. Hence, the mechanism of the interactions among oxidative stress, inflammation, and ER stress in the development of depression requires further investigation.

\section{Acknowledgements}

Not applicable.

\section{Funding}

The present study was funded by The Medical and Health Science and Technology Plan Project of Zhejiang Province (grant nos. 2018KY671 and 2019KY564), The Ningbo Health Branding Subject Fund (grant no. PPXK2018-01), The Major Social Development Special Foundation of Ningbo (grant no. 2017C510010), The Natural Science Foundation of Zhejiang province (grant no. LY14H090003) and The Natural Science Foundation of Ningbo (grant no. 2016A610156).

\section{Availability of data and materials}

Not applicable. 


\section{Authors' contributions}

JXM, YRH and ZZL drafted the manuscript. JXM, YXJ and ZZL conceived and designed the framework of this article, LMR, YRH, YXJ and ZZL collected and analyzed the literature. All authors read and approved the final manuscript.

\section{Ethics approval and consent to participate}

Not applicable.

\section{Patient consent for publication}

Not applicable.

\section{Competing interests}

The authors declare that they have no competing interests.

\section{References}

1. Malhi GS and Mann JJ: Depression. Lancet (London, England) 392: 2299-2312, 2018.

2. The burden of depression. Nature 515: 163, 2014.

3. Ledford H: Medical research: If depression were cancer. Nature 515: 182-184, 2014

4. McCaffrey K and Braakman I: Protein quality control at the endoplasmic reticulum. Essays Biochem 60: 227-235, 2016.

5. Hetz C and Papa FR: The unfolded protein response and cell fate control. Mol Cell 69: 169-181, 2018.

6. Hetz C, Chevet E and Oakes SA: Proteostasis control by the unfolded protein response. Nat Cell Biol 17: 829-838, 2015.

7. Wang J, Lee J, Liem D and Ping P: HSPA5 Gene encoding Hsp70 chaperone $\mathrm{BiP}$ in the endoplasmic reticulum. Gene 618: 14-23, 2017.

8. Behnke J, Mann MJ, Scruggs FL, Feige MJ and Hendershot LM: Members of the Hsp70 family recognize distinct types of sequences to execute ER quality control. Mol Cell 63: 739-752, 2016.

9. Otero JH, Lizák B and Hendershot LM: Life and death of a BiP substrate. Semin Cell Dev Biol 21: 472-478, 2010.

10. Li J, Ni M, Lee B, Barron E, Hinton DR and Lee AS: The unfolded protein response regulator $\mathrm{GRP} 78 / \mathrm{BiP}$ is required for endoplasmic reticulum integrity and stress-induced autophagy in mammalian cells. Cell Death Differ 15: 1460-1471, 2008.

11. Nakamura K, Bossy-Wetzel E, Burns K, Fadel MP, Lozyk M Goping IS, Opas M, Bleackley RC, Green DR and Michalak M: Changes in endoplasmic reticulum luminal environment affect cell sensitivity to apoptosis. J Cell Biol 150: 731-740, 2000.

12. Bown C, Wang JF, MacQueen G and Young LT: Increased temporal cortex ER stress proteins in depressed subjects who died by suicide. Neuropsychopharmacology 22: 327-332, 2000.

13. Nevell L, Zhang K, Aiello AE, Koenen K, Galea S, Soliven R, Zhang C, Wildman DE and Uddin M: Elevated systemic expression of ER stress related genes is associated with stress-related mental disorders in the Detroit Neighborhood Health Study. Psychoneuroendocrinology 43: 62-70, 2014.

14. Koenig JI, Walker CD, Romeo RD and Lupien SJ: Effects of stress across the lifespan. Stress 14: 475-480,2011.

15. Dillon DG and Pizzagalli DA: Mechanisms of memory disruption in depression. Trends Neurosci 41: 137-149, 2018.

16. Kadowaki H, Satrimafitrah P, Takami Y and Nishitoh $\mathrm{H}$ : Molecular mechanism of ER stress-induced pre-emptive quality control involving association of the translocon, Derlin-1, and HRD1. Sci Rep 8: 7317, 2018

17. Oakes SA and Papa FR: The role of endoplasmic reticulum stress in human pathology. Annu Rev Pathol 10: 173-194, 2015.

18. Shore GC, Papa FR and Oakes SA: Signaling cell death from the endoplasmic reticulum stress response. Curr Opin Cell Biol 23: 143-149, 2011.

19. Scheper W and Hoozemans JJ: The unfolded protein response in neurodegenerative diseases: A neuropathological perspective. Acta Neuropathol 130: 315-331, 2015.
20. Bettigole SE and Glimcher LH: Endoplasmic reticulum stress in immunity. Annu Rev Immunol 33: 107-138, 2015.

21. Korennykh A and Walter P: Structural basis of the unfolded protein response. Annu Rev Cell Dev Biol 28: 251-277, 2012.

22. Casas C: GRP78 at the centre of the stage in cancer and neuroprotection. Front Neurosci 11: 177, 2017.

23. Harding HP, Zhang Y, Bertolotti A, Zeng H and Ron D: Perk is essential for translational regulation and cell survival during the unfolded protein response. Mol Cell 5: 897-904, 2000.

24. Carrara M, Prischi F, Nowak PR, Kopp MC and Ali MM: Noncanonical binding of BiP ATPase domain to Irel and Perk is dissociated by unfolded protein $\mathrm{CH} 1$ to initiate ER stress signaling. Elife 4, 2015.

25. Han J, Back SH, Hur J, Lin YH, Gildersleeve R, Shan J, Yuan CL, Krokowski D, Wang S, Hatzoglou M, et al: ER-stress-induced transcriptional regulation increases protein synthesis leading to cell death. Nat Cell Biol 15: 481-490, 2013.

26. Yang J, Liu H, Li L, Liu H, Shi W, Yuan X and Wu L: Structural insights into IRE1 functions in the unfolded protein response. Curr Med Chem 23: 4706-4716, 2016.

27. Lee AH, Iwakoshi NN and Glimcher LH: XBP-1 regulates a subset of endoplasmic reticulum resident chaperone genes in the unfolded protein response. Mol Cell Biol 23: 7448-7459, 2003.

28. Wu R, Zhang QH, Lu YJ, Ren K and Yi GH: Involvement of the IRE1 $\alpha$-XBP1 pathway and XBP1s-dependent transcriptional reprogramming in metabolic diseases. DNA Cell Biol 34: 6-18, 2015.

29. Papaioannou A, Higa A, Jégou G, Jouan F, Pineau R, Saas L, Avril T, Pluquet $\mathrm{O}$ and Chevet E: Alterations of EDEM1 functions enhance ATF6 pro-survival signaling. FEBS J 285: 4146-4164, 2018

30. Hillary RF and FitzGerald U: A lifetime of stress: ATF6 in development and homeostasis. J Biomed Sci 25: 48, 2018

31. Shoulders MD, Ryno LM, Genereux JC, Moresco JJ, Tu PG, Wu C, Yates JR III, Su AI, Kelly JW and Wiseman RL: Stress-independent activation of XBP1s and/or ATF6 reveals three functionally diverse ER proteostasis environments. Cell Rep 3: 1279-1292, 2013.

32. Hiramatsu N, Chiang WC, Kurt TD, Sigurdson CJ and Lin JH: Multiple mechanisms of unfolded protein response-induced cell death. Am J Pathol 185: 1800-1808, 2015.

33. Hill MN, Hellemans KG, Verma P, Gorzalka BB and Weinberg J: Neurobiology of chronic mild stress: Parallels to major depression. Neurosci Biobehav Rev 36: 2085-2117, 2012.

34. Liu Y, Yang N, Hao W, Zhao Q, Ying T, Liu S, Li Q, Liang Y, Wang T, Dong Y, et al: Dynamic proteomic analysis of protein expression profiles in whole brain of Balb/C mice subjected to unpredictable chronic mild stress: Implications for depressive disorders and future therapies. Neurochem Int 58: 904-913, 2011.

35. Tan H, Zou W, Jiang J, Tian Y, Xiao Z, Bi L, Zeng H and Tang X Disturbance of hippocampal H2S generation contributes to CUMS-induced depression-like behavior: Involvement in endoplasmic reticulum stress of hippocampus. Acta Biochim Biophys Sin (Shanghai) 47: 285-291, 2015.

36. Zhang $X$ and Bian JS: Hydrogen sulfide: A neuromodulator and neuroprotectant in the central nervous system. ACS Chem Neurosci 5: 876-883, 2014.

37. Liu SY, Li D, Zeng HY, Kan LY, Zou W, Zhang P, Gu HF and Tang XQ: Hydrogen sulfide inhibits chronic unpredictable mild stress-induced depressive-like behavior by upregulation of Sirt-1: Involvement in suppression of hippocampal endoplasmic reticulum stress. Int J Neuropsychopharmacol 20: 867-876, 2017.

38. Chiba S, Numakawa T, Ninomiya M, Richards MC, Wakabayashi $\mathrm{C}$ and Kunugi $\mathrm{H}$ : Chronic restraint stress causes anxiety- and depression-like behaviors, downregulates glucocorticoid receptor expression, and attenuates glutamate release induced by brain-derived neurotrophic factor in the prefrontal cortex. Prog Neuropsychopharmacol Biol Psychiatry 39: 112-119, 2012.

39. Jangra A, Dwivedi S, Sriram CS, Gurjar SS, Kwatra M, Sulakhiya K, Baruah CC and Lahkar M: Honokiol abrogates chronic restraint stress-induced cognitive impairment and depressive-like behaviour by blocking endoplasmic reticulum stress in the hippocampus of mice. Eur J Pharmacol 770: 25-32, 2016.

40. Jangra A, Sriram CS, Dwivedi S, Gurjar SS, Hussain MI, Borah P and Lahkar M: Sodium phenylbutyrate and edaravone abrogate chronic restraint stress-induced behavioral deficits: Implication of oxido-nitrosative, endoplasmic reticulum stress cascade, and neuroinflammation. Cell Mol Neurobiol 37: 65-81, 2017. 
41. Pavlovsky AA, Boehning D, Li D, Zhang Y, Fan X and Green TA: Psychological stress, cocaine and natural reward each induce endoplasmic reticulum stress genes in rat brain. Neuroscience 246: 160-169, 2013.

42. Lawson MA, Parrott JM, McCusker RH, Dantzer R, Kelley KW and O'Connor JC: Intracerebroventricular administration of lipopolysaccharide induces indoleamine-2,3-dioxygenase-dependent depression-like behaviors. J Neuroinflammation 10: 87, 2013.

43. Jangra A, Sriram CS and Lahkar M: Lipopolysaccharide-induced behavioral alterations are alleviated by sodium phenylbutyrate via attenuation of oxidative stress and neuroinflammatory cascade. Inflammation 39: 1441-1452, 2016

44. Huang GB, Zhao T, Muna SS, Bagalkot TR, Jin HM, Chae HJ and Chung YC: Effects of chronic social defeat stress on behaviour endoplasmic reticulum proteins and choline acetyltransferase in adolescent mice. Int J Neuropsychopharmacol 16: 1635-1647, 2013.

45. Liu L, Zhao Z, Lu L, Liu J, Sun J, Wu X and Dong J: Icariin and icaritin ameliorated hippocampus neuroinflammation via inhibiting HMGB1-related pro-inflammatory signals in lipopolysaccharide-induced inflammation model in C57BL/6J mice. Int Immunopharmacol 68: 95-105, 2019.

46. Li MX, Li Q, Sun XJ, Luo C, Li Y, Wang YN, Chen J, Gong CZ, Li YJ, Shi LP, et al: Increased Homer1-mGluR5 mediates chronic stress-induced depressive-like behaviors and glutamatergic dysregulation via activation of PERK-eIF2 $\alpha$. Prog Neuropsychopharmacol Biol Psychiatry 95: 109682, 2019.

47. Sharma V, Ounallah-Saad H, Chakraborty D, Hleihil M, Sood R, Barrera I, Edry E, Kolatt Chandran S, Ben Tabou de Leon S, Kaphzan H and Rosenblum K: Local inhibition of PERK enhances memory and reverses age-related deterioration of cognitive and neuronal properties. J Neurosci 38: 648-658, 2018.

48. Timberlake MA II and Dwivedi Y: Altered expression of endoplasmic reticulum stress associated genes in hippocampus of learned helpless rats: Relevance to depression pathophysiology. Front Pharmacol 6: 319, 2016.

49. Luedtke K, Bouchard SM, Woller SA, Funk MK, Aceves M and Hook MA: Assessment of depression in a rodent model of spinal cord injury. J Neurotrauma 31: 1107-1121, 2014.

50. Maldonado-Bouchard S, Peters K, Woller SA, Madahian B Faghihi U, Patel S, Bake S and Hook MA: Inflammation is increased with anxiety- and depression-like signs in a rat model of spinal cord injury. Brain Behav Immun 51: 176-195, 2016.

51. Wu J, Zhao Z, Sabirzhanov B, Stoica BA, Kumar A, Luo T, Skovira J and Faden AI: Spinal cord injury causes brain inflammation associated with cognitive and affective changes: Role of cell cycle pathways. J Neurosci 34: 10989-11006, 2014.

52. Wu J, Zhao Z, Kumar A, Lipinski MM, Loane DJ, Stoica BA and Faden AI: Endoplasmic reticulum stress and disrupted neurogenesis in the brain are associated with cognitive impairment and depressive-like behavior after spinal cord injury. J Neurotrauma 33: 1919-1935, 2016.

53. Cheng Y, Cawley NX, Yanik T, Murthy SR, Liu C, Kasikci F, Abebe D and Loh YP: A human carboxypeptidase E/NF- $\alpha 1$ gene mutation in an Alzheimer's disease patient leads to dementia and depression in mice. Transl Psychiatry 6: e973, 2016.

54. Michel TM, Camara S, Tatschner T, Frangou S, Sheldrick AJ, Riederer P and Grünblatt E: Increased xanthine oxidase in the thalamus and putamen in depression. World J Biol Psychiatry 11: 314-320, 2010.

55. Harrison R: Structure and function of xanthine oxidoreductase: Where are we now? Free Radic Biol Med 33: 774-797, 2002.

56. Ishisaka M, Kakefuda K, Yamauchi M, Tsuruma K, Shimazawa M, Tsuruta A and Hara H: Luteolin shows an antidepressant-like effect via suppressing endoplasmic reticulum stress. Biol Pharm Bull 34: 1481-1486, 2011

57. Liu Y, Zhao N, Li C, Chang Q, Liu X, Liao Y and Pan R: Longistyline $\mathrm{C}$ acts antidepressant in vivo and neuroprotection in vitro against glutamate-induced cytotoxicity by regulating NMDAR/NR2B-ERK pathway in PC12 cells. PLoS One 12: e0183702, 2017.

58. Jiang Y, Li Z, Liu Y, Liu X, Chang Q, Liao Y and Pan R: Neuroprotective effect of water extract of Panax ginseng on corticosterone-induced apoptosis in PC12 cells and its underlying molecule mechanisms. J Ethnopharmacol 159: 102-112, 2015.

59. Li ZY, Guo Z, Liu YM, Liu XM, Chang Q, Liao YH and Pan RL: Neuroprotective effects of total saikosaponins of Bupleurum yinchowense on corticosterone-induced apoptosis in PC12 cells J Ethnopharmacol 148: 794-803, 2013.
60. Ye T, Meng X, Wang R, Zhang C, He S, Sun G and Sun X: Gastrodin alleviates cognitive dysfunction and depressive-like behaviors by inhibiting ER stress and NLRP3 inflammasome activation in db/db Mice. Int J Mol Sci 19, 2018.

61. Shikov AN, Pozharitskaya ON and Makarov VG: Aralia elata var. mandshurica (Rupr. \& Maxim.) J.Wen: An overview of pharmacological studies. Phytomedicine 23: 1409-1421, 2016.

62. Narita N, Hashimoto K. Tomitaka S and Minabe Y: Interactions of selective serotonin reuptake inhibitors with subtypes of sigma receptors in rat brain. Eur J Pharmacol 307: 117-119, 1996.

63. Hosoi T, Miyahara T, Kayano T, Yokoyama S and Ozawa K Fluvoxamine attenuated endoplasmic reticulum stress-induced leptin resistance. Front Endocrinol (Lausanne) 3: 12, 2012.

64. Hayashi T and Su TP: Sigma-1 receptor chaperones at the ER-mitochondrion interface regulate $\mathrm{Ca}(2+)$ signaling and cell survival. Cell 131: 596-610, 2007.

65. Omi T, Tanimukai H, Kanayama D, Sakagami Y, Tagami S, Okochi M, Morihara T, Sato M, Yanagida K, Kitasyoji A, et al: Fluvoxamine alleviates ER stress via induction of Sigma-1 receptor. Cell Death Dis 5: e1332, 2014.

66. Terada K, Izumo N, Suzuki B, Karube Y, Morikawa T, Ishibashi Y, Kameyama T, Chiba K, Sasaki N, Iwata K, et al: Fluvoxamine moderates reduced voluntary activity following chronic dexamethasone infusion in mice via recovery of BDNF signal cascades. Neurochem Int 69: 9-13, 2014.

67. Perez-Caballero L, Torres-Sanchez S, Bravo L, Mico JA and Berrocoso E: Fluoxetine: A case history of its discovery and preclinical development. Expert Opin Drug Discov 9: 567-578, 2014.

68. Ma J, Yang YR, Chen W, Chen MH, Wang H, Wang XD, Sun LL, Wang FZ and Wang DC: Fluoxetine synergizes with temozolomide to induce the CHOP-dependent endoplasmic reticulum stress-related apoptosis pathway in glioma cells. Oncol Rep 36: 676-684, 2016.

69. Ma J, Hou LN, Rong ZX, Liang P, Fang C, Li HF, Qi H and Chen HZ: Antidepressant desipramine leads to C6 glioma cell autophagy: Implication for the adjuvant therapy of cancer. Anticancer Agents Med Chem 13: 254-260, 2013.

70. Abelaira HM, Réus GZ, Ignácio ZM, Dos Santos MA, de Moura AB, Matos D, Demo JP, da Silva JB, Michels M, Abatti M, et al: Effects of ketamine administration on mTOR and reticulum stress signaling pathways in the brain after the infusion of rapamycin into prefrontal cortex. J Psychiatr Res 87: 81-87, 2017.

71. Chen S, Xuan J, Couch L, Iyer A, Wu Y, Li QZ and Guo L: Sertraline induces endoplasmic reticulum stress in hepatic cells. Toxicology 322: 78-88, 2014.

72. Ren Z, Chen S, Zhang J, Doshi U, Li AP and Guo L: Endoplasmic reticulum stress induction and ERK1/2 activation contribute to nefazodone-induced toxicity in hepatic cells. Toxicol Sci 154: 368-380, 2016.

73. Yang L, Zheng L, Wan Y, Chen Z, Li P and Wang Y: Metoprolol, $\mathrm{N}$-acetylcysteine, and escitalopram prevents chronic unpredictable mild stress-induced depression by inhibition of endoplasmic reticulum stress. Front Psychiatry 9: 696, 2018.

74. Li B, Yadav RK, Jeong GS, Kim HR and Chae HJ: The characteristics of Bax inhibitor-1 and its related diseases. Curr Mol Med 14: 603-615, 2014.

75. Sui ZY, Chae HJ, Huang GB, Zhao T, Shrestha Muna S and Chung YC: Effects of chronic mild stress in female bax inhibitor-1-gene knockout mice. Clin Psychopharmacol Neurosci 10: $155-162,2012$.

76. Hunsberger JG, Machado-Vieira R, Austin DR, Zarate C, Chuang DM, Chen G, Reed JC and Manji HK: Bax inhibitor 1, a modulator of calcium homeostasis, confers affective resilience. Brain Res 1403: 19-27, 2011.

77. Crider A, Nelson T, Davis T, Fagan K, Vaibhav K, Luo M, Kamalasanan S, Terry AV Jr and Pillai A: Estrogen receptor $\beta$ agonist attenuates endoplasmic reticulum stress-induced changes in social behavior and brain connectivity in mice. Mol Neurobiol 55: 7606-7618, 2018

78. Swardfager W, Hennebelle M, Yu D, Hammock BD, Levitt AJ, Hashimoto K and Taha AY: Metabolic/inflammatory/vascular comorbidity in psychiatric disorders; soluble epoxide hydrolase (sEH) as a possible new target. Neurosci Biobehav Rev 87: 56-66, 2018 .

This work is licensed under a Creative Commons Attribution-NonCommercial-NoDerivatives 4.0 International (CC BY-NC-ND 4.0) License. 